\title{
Types of inferior mesenteric artery: a proposal for a new classification
}

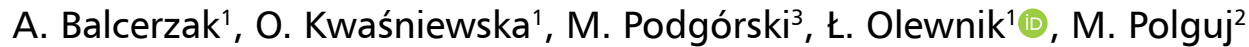 \\ ${ }^{1}$ Department of Anatomical Dissection and Donation, Medical University of Lodz, Poland \\ 2Department of Normal and Clinical Anatomy, Chair of Anatomy and Histology, Medical University of Lodz, Poland \\ ${ }^{3}$ Polish Mother's Memorial Hospital Research Institute, Lodz, Poland
}

[Received: 23 June 2020; Accepted: 26 August 2020; Early publication date: 15 September 2020]

Background: The inferior mesenteric artery (IMA) is the third main branch of the abdominal aorta and arises at the level of L3, supplying the large intestine from the distal transverse colon to the upper part of the anal canal. The aim of this study was to characterise the course and morphology of the terminal branches of the IMA, and also creating a new IMA classification, which seems to be necessary for clinicians performing surgery in this area.

Materials and methods: The anatomical variations in the branching patterns of the IMA were examined in 40 cadavers fixed in a $10 \%$ formalin solution. Morphometric measurements were then obtained twice by two researchers.

Results: Due to the proposed classification system, type I characterised by a common trunk preceding bifurcation into sigmoidal branches and the superior rectal artery after giving left colic artery (LCA) occurred in 57.5\% of cases, type II, trifurcation type, in 25\%, type III with the superior rectal arteries originating firstly from the IMA in 5\%, type IV with the ascending lumbar artery in 10\% of cases and type $V$, a novelty, in $2.5 \%$. The origin of IMA was observed at the level of $L 2 / L 3$ in $22.5 \%$ of cases, at $L 3$ in $25 \%$ of cases, at $L 3 / L 4$ in $15 \%$ of cases, at $L 4$ in $35 \%$ of cases and at the level of $L 5$ in $2.5 \%$ of cases. More than one third (38.1\%) of total cases with additional arteries and rapidly bifurcating branches occurred in types III, IV, and V. In women, only the IMA and LCA were significantly narrower than in men. Only the diameter of the IMA correlated with the diameter of the superior rectal artery. Conclusions: The IMA is characterised by high morphological variability. The introduction of a new, structured, anatomical classification seems necessary for all clinicians. (Folia Morphol 2021; 80, 4: 827-838)

Key words: anatomical variations, inferior mesenteric artery, left colic artery, superior rectal artery, sigmoid branches, new classification, cadavers

\section{INTRODUCTION}

The inferior mesenteric artery (IMA) is the third main branch of the abdominal aorta and normally arises at the level of L3. It transports and supplies arterial blood to a significant portion of the gastrointestinal tract - the distal 1/3 of the transverse colon, splenic flexure, descending colon, sigmoid colon and rectum [14]. 
The most common branches of the IMA include: 1) the left colic, which is located retroperitoneally and supplies the descending colon; 2) the sigmoid branches, which supply the sigmoid colon; and 3) the superior rectal artery or arteries, which are the terminal branches of the IMA at the pelvic brim. Nonetheless, the IMA shows considerable anatomic variations. There have been reports of the left colic artery arising from the abdominal aorta, the IMA giving a branch to the left kidney or the left renal artery arising from the IMA and also a lot of other case reports, which describe numerous variations $[1,3,7,9,12,13,15]$.

Familiarity with anatomical variations of the IMA is relevant to planning radiological and surgical procedures in the distal 1/3 part of the transverse colon, splenic flexure, descending colon, sigmoid colon, rectum and also in any deeply reaching interventions in the left iliac and hypogastrium region like radical resection with preservation of the left colic artery for rectal cancer or embolisation [24]. Embolisation is a remarkably versatile procedure used in nearly all vascular and nonvascular systems to treat a wide range of pathology. Embolisation is a minimally invasive surgical technique. The purpose is to close the lumen of the artery and prevent blood flow to an area of the body, which can effectively shrink a tumour or block an aneurysm.

The aim of our research is to determine variations of the branch origin from the IMA and its anatomical variations, including the occurrence of any additional arteries in the Caucasian population, which may be relevant to minimising complications after diagnostic tests and surgical procedures. A novelty in this work is a new, structured, anatomical classification that includes new types of IMA branches and multiple additional arteries, which are associated with more frequent selective endovascular application of chemotherapeutics to tumours of the transverse, descending or sigmoid colon, rectum, surgical removal of its parts and also selective embolisation. We hypothesize that the IMA is characterised by high morphological variability.

\section{MATERIALS AND METHODS}

Forty cadavers, including 21 male (M) and 19 female (F) cadavers, were studied. They were fixed in a $10 \%$ formalin solution. The specimens had no traces of surgical intervention in the abdominal area. The Local Bioethics Commission issued a consent for the study (consent no. RNN/93/16/KE).
The study was carried out with classical dissection techniques [17-20]. During the dissection, the IMA morphology, the point where it branched off the abdominal aorta in reference to the spine, the presence of additional arterial branches and of quickly-bifurcating arteries, i.e. those shorter than $2 \mathrm{~cm}$, were evaluated.

The next stage consisted in morphometric measurements of the distance between the origin of IMA and first bifurcation, between the first bifurcation and the second, and between the common trunks from which the final branches originated. Each arterial diameter measurement was taken at the point of its origin from the appropriate artery.

The measurements were taken with an electronic calliper (Mitutoyo Corporation, Kawasaki-shi, Kanagawa, Japan). Each measurement was taken independently twice by two people with high experience in anatomical dissection, accurate within $0.1 \mathrm{~mm}$ as in many other studies $[16,17,20]$.

\section{Ethical approval and consent to participate}

The anatomical protocol of the study was accepted by the Bioethics Committee of the Medical University of Lodz (resolution consent no. RNN/93/16/KE). The cadavers belong to the Department of Anatomical Dissection and Donation of the Medical University of Lodz, Poland.

\section{Statistical analysis}

The type of IMA was compared between sexes with the $\chi^{2}$ test. The normality of the diameter distribution was checked with the Shapiro-Wilk test. As the data was not normally distributed in further comparisons, non-parametric tests were used (the Mann-Whitney, the Wilcoxon and the Kruskal-Wallis ANOVA with a dedicated post hoc test). Correlation was assessed with the Spearman's rank coefficient. Analysis was performed with the Statistica 13 software (StatSoft Polska, Krakow, Poland). A p-value lower than 0.05 was considered significant, and the results are presented as mean and standard deviation unless otherwise stated.

\section{RESULTS}

\section{Evaluation of branching patterns of the IMA}

Five main types of branching were distinguished based on the morphology of the IMA. Additionally, type I and type II were subdivided into subtype IA, subtype IB, subtype IC, subtype IIA and subtype IIB. 


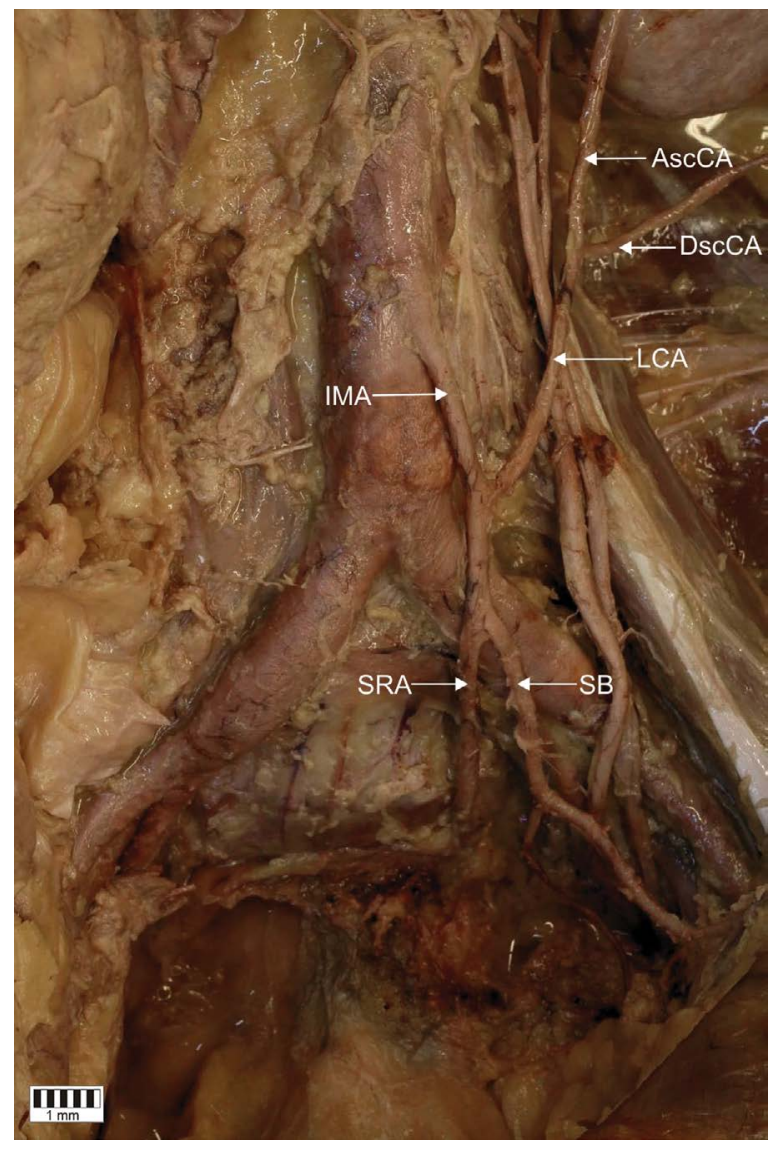

Figure 1. Inferior mesenteric artery (IMA) branching type IA. Independent origin of left colic artery (LCA), which further divides into ascending colic artery (AscCA) and descending colic artery (DscCA); SB — sigmoidal branch; SRA — superior rectal artery.

- Type I - exemplified by the presence of a common trunk which bifurcates into a sigmoidal branch and the superior rectal artery after giving the left colic artery (LCA) or ascending and descending left colic arteries, with the LCA itself being absent. Three subtypes were determined based on different originating and branching patterns of LCA. This type was present in 23 (57.5\%) cases.

- Type IA - an independent origin of the LCA from the IMA; a common trunk preceding bifurcation into the ascending and descending left colic arteries. An independent origin of the sigmoid branch/branches and superior rectal artery. This subtype was present in $10(25 \%)$ cases (Fig. 1).

- Type IB - an independent origin of the LCA from the IMA; no bifurcation observed. An independent origin of the sigmoid branch/branches and superior rectal artery (SRA). This subtype was observed in $8(20 \%)$ cases (Fig. 2).

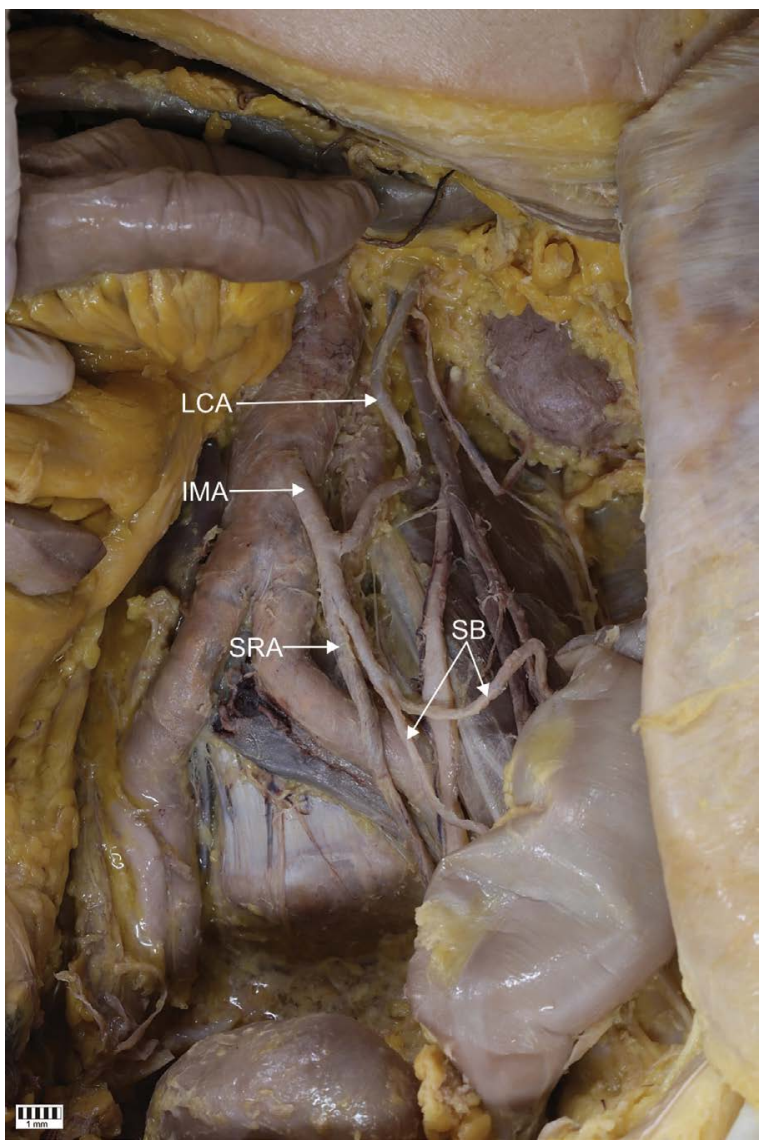

Figure 2. Inferior mesenteric artery (IMA) type IB. Independent origin of left colic artery (LCA), no bifurcation; SB — sigmoidal branches; SRA — superior rectal artery.

- Type IC - independent origins of the branches of LCA - ascending and descending left colic arteries; no common trunk preceding. An independent origin of the sigmoid branch/branches and superior rectal artery. This subtype was present in $5(12.5 \%)$ cases (Fig. 3).

- Type II - trifurcation type - two subtypes were determined based on different originating and branching patterns of the LCA. This type was observed in $10(25 \%)$ cases.

- Type IIA - the LCA; bifurcation into the left ascending colic artery (AscCA) and left descending colic artery (DscCA) preceded by the common trunk, sigmoid branch/branches and SRA originating from trifurcation of IMA. This subtype was observed in $8(20 \%)$ cases (Fig. 4).

- Type IIB - the LCA; without bifurcation into the ascending and descending arteries, sigmoid branch/branches and the SRA originat- 


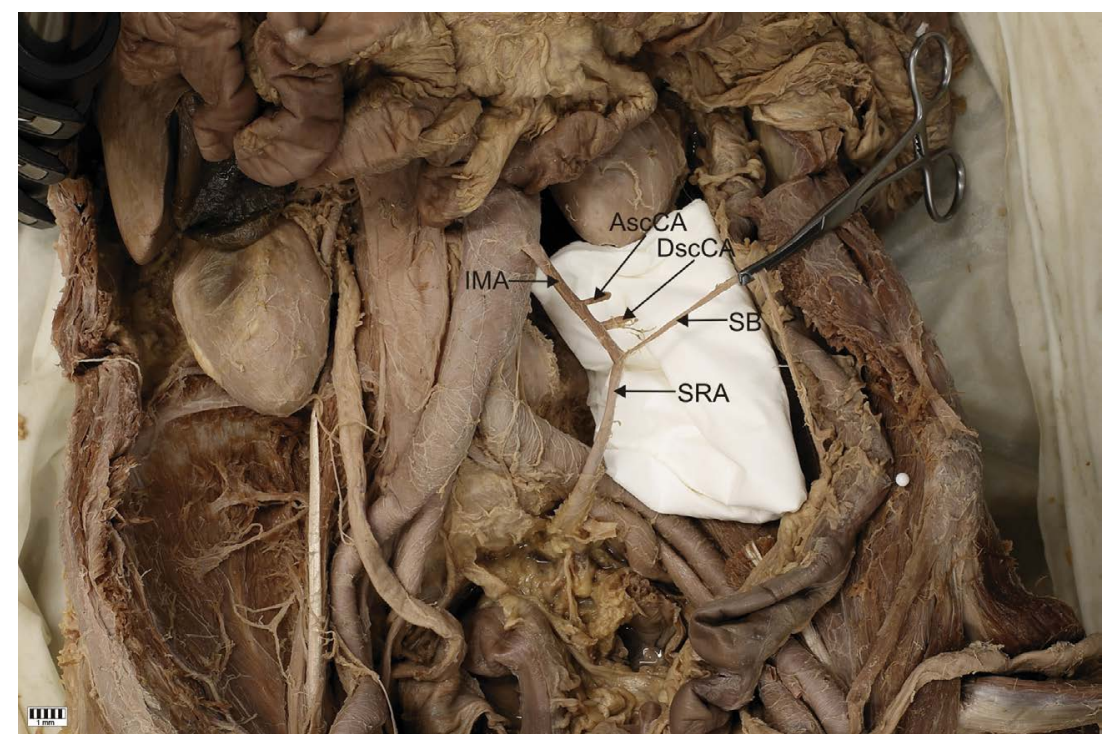

Figure 3. Inferior mesenteric artery (IMA) branching type IC. Lack of left colic artery, ascending colic artery (AscCA) and descending colic artery (DscCA) originating directly from IMA; SB — sigmoidal branch; SRA — superior rectal artery.

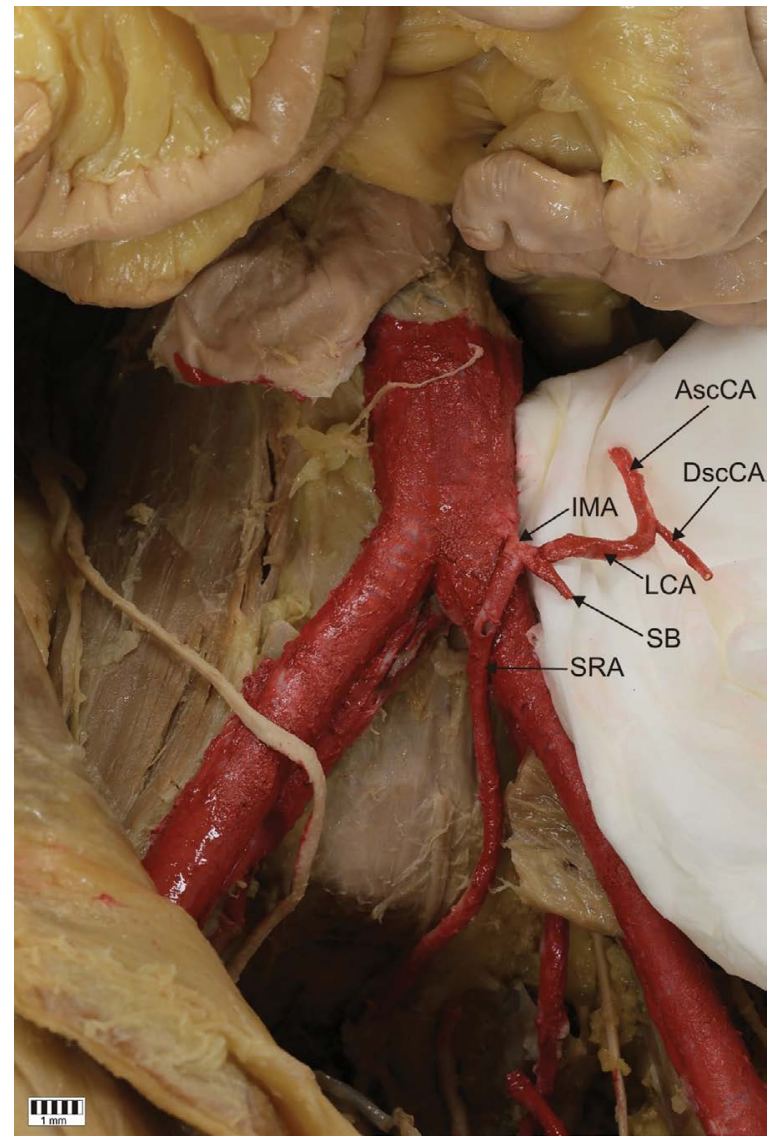

Figure 4. Inferior mesenteric artery (IMA) branching type IIA. Trifurcation type with left colic artery (LCA) branching off ascending colic artery (AscCA) and descending colic artery (DscCA); SB sigmoidal branch; SRA — superior rectal artery.

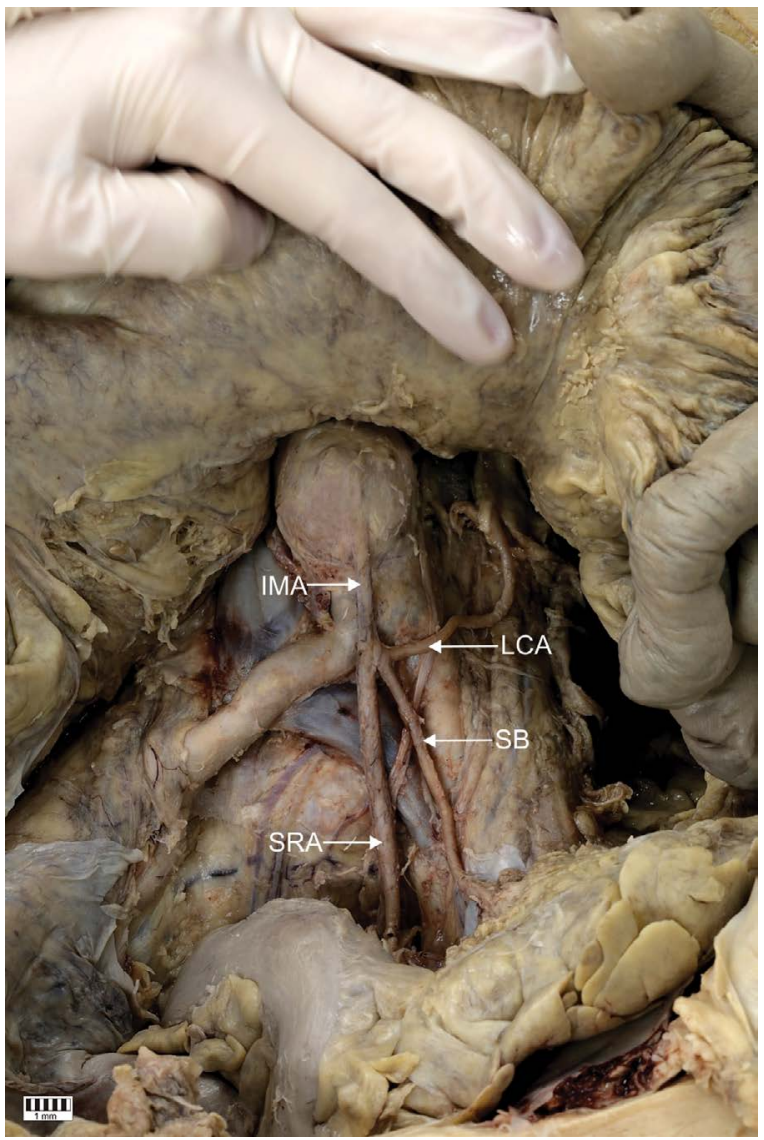

Figure 5. Inferior mesenteric artery (IMA) branching type IIB trifurcation type with absence of left colic artery (LCA) bifurcation; SB — sigmoidal branch; SRA — superior rectal artery. 


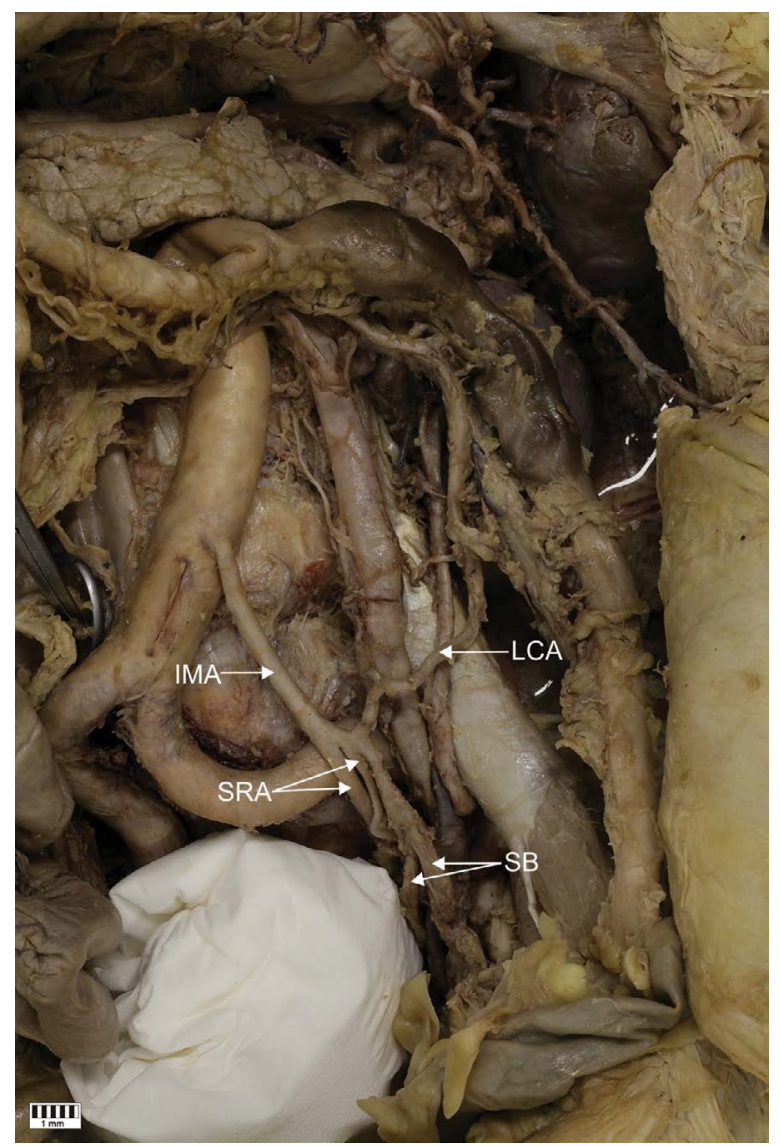

Figure 6. Inferior mesenteric artery (IMA) branching type III superior rectal arteries (SRA) originating firstly; LCA — left colic artery; SB — sigmoidal branches.

ing from trifurcation of IMA. This subtype was present in 2 (5\%) cases (Fig. 5).

- Type III - the superior rectal artery (arteries) originating firstly from the IMA, the common trunk preceding bifurcation into the sigmoidal branch/branches (SB) and LCA. This type was present in $2(5 \%)$ cases. In both of them, two SRAs were observed instead of a single one and, to our best knowledge, this branching pattern has not been previously observed in studies on IMA (Fig. 6).

- Type IV - the ascending lumbar artery originating independently from the IMA, the common trunk dividing into the LCA and main SB, second sigmoid branch and SRA originating independently from the IMA. To our knowledge, this type was observed in $4(10 \%)$ cases. This branching pattern has not been previously classified by other authors (Fig. 7).

- Type $\mathrm{V}$ - the ascending LCA originating independently from the IMA. The first bifurcation of IMA divides it into the common superior rectal trunk and the common trunk branching off into

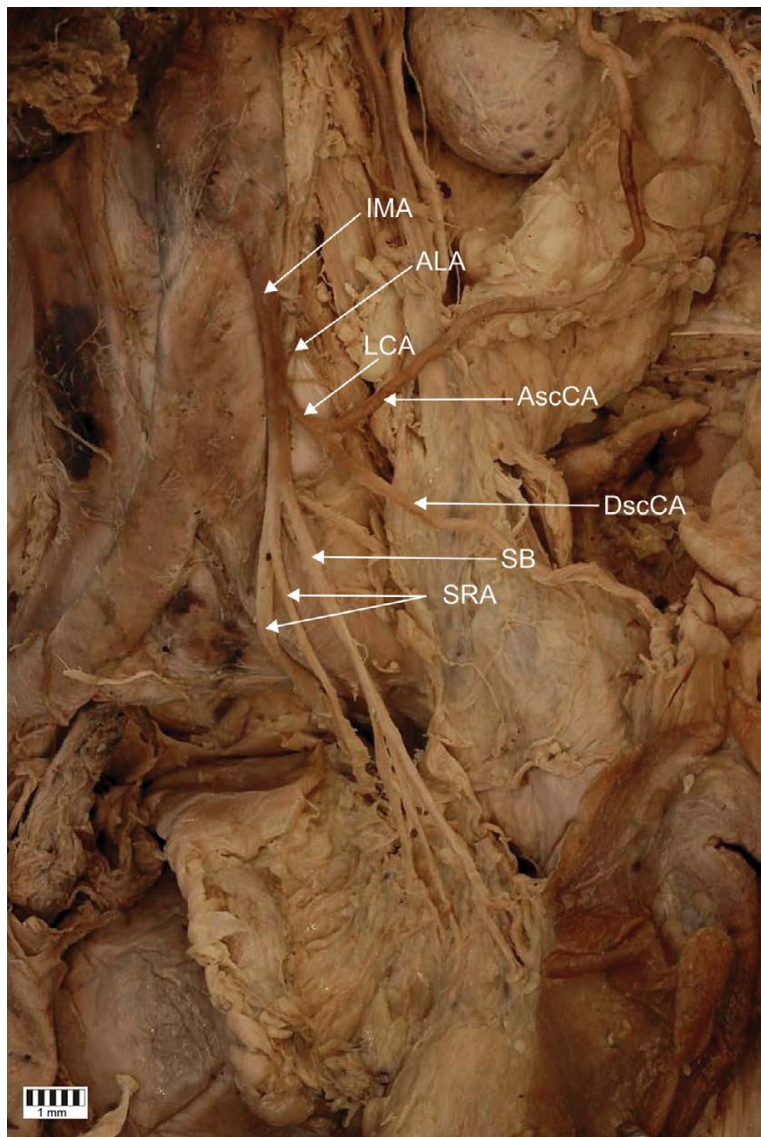

Figure 7. Inferior mesenteric artery (IMA) branching type IV ascending lumbar artery (ALA) originating from IMA; LCA — left colic artery; AscCA - ascending colic artery; DscCA - descending colic artery; SB — sigmoidal branch; SRA — superior rectal arteries.

the descending LCA and the common trunk for sigmoidal branches (2). The ascending and descending left colic arteries create an anastomose, which has a further connection with the marginal artery of Drummond upwards, and with sigmoidal branches downwards. This type was present only in $1(2.5 \%)$ case. To our best knowledge, this branching pattern has not been previously classified by other authors (Fig. 8).

\section{Evaluation of the level of the IMA origin}

The study revealed five different variations of the level of the IMA origin:

1. At the level of the intervertebral disc between the second and third lumbar vertebra $-22.5 \%$ (9/40) observed respectively:

- in type I $-21.7 \%$ of cases $(5 / 23)$;

- in type II - $20 \%$ of cases (2/10);

- in type III - $50 \%$ of cases (1/2);

- in type IV $-25 \%$ of cases (1/4). 


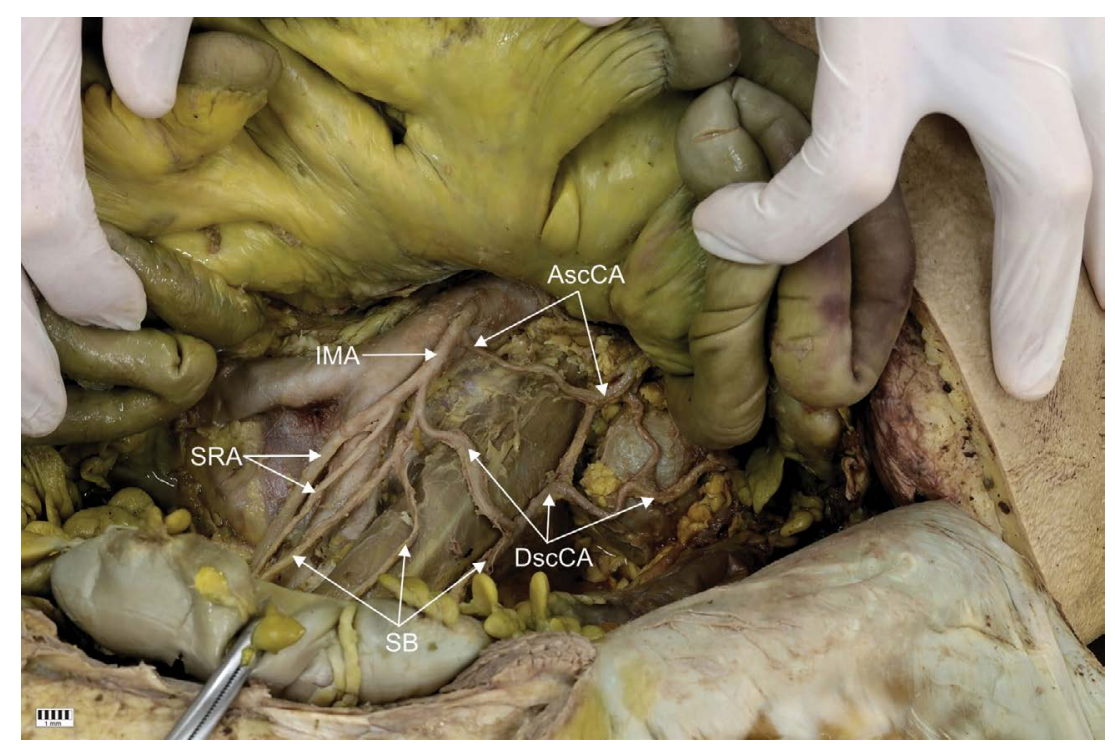

Figure 8. Inferior mesenteric artery (IMA) type $V$ — rare case of IMA branching off ascending colic artery (AscCA) firstly, which further anastomose multiple times with descending colic artery (DscCA), DscCA and sigmoidal branch, which further divides, originating from IMA bifurcation; SRA — superior rectal arteries; SB — sigmoidal branches.

2. At the level of the third lumbar vertebra $-25 \%$ (10/40):

- in type I $-26.1 \%$ of cases (6/23);

- in type II - $30 \%$ of cases $(3 / 10)$;

- in type IV $-25 \%$ of cases (1/4).

3. At the level of the intervertebral disc between the third and fourth lumbar vertebra $-15 \%$ $(6 / 40)$.

- in type I - $13 \%$ of cases (3/23);

- in type II $-10 \%$ of cases $(1 / 10)$;

- in type IV $-25 \%$ of cases (1/4).

4. At the level of the fourth lumbar vertebra $-35 \%$

(14/40).

- in type I $-34.8 \%$ of cases (8/23);

- in type II $-40 \%$ of cases $(4 / 10)$;

- in type III $-50 \%$ of cases $(1 / 2)$;

- in type $V-100 \%$ of cases (1/1).

5. At the level of the fifth lumbar vertebra $-2.5 \%$ $(1 / 40)$, which, to our best knowledge, is a case that has never been reported before - observed in type I of the branching pattern.

\section{Evaluation of the occurrence of additional SBs or SRAs}

The study revealed:

1. The occurrence of more than one sigmoidal branch - observed in nine (22.5\%) cases.

- type I - in 3 cases, which is $33.3 \%(3 / 9)$;

- type III - in 2 cases, which is $22.2 \%$ (2/9);

- type IV - in 4 cases, which is $22.2 \%$ (4/9).
2. The occurrence of the quickly bifurcating sigmoidal branch - observed in $5(12.5 \%)$ cases.

- type I - in 3 cases, which is $60 \%(3 / 5)$;

- type II - in 1 case, which is $20 \%(1 / 5)$;

- type V - in 1 case, which is $20 \%(1 / 5)$.

3. The occurrence of more than one superior rectal artery - present in $6(15 \%)$ cases.

- type I - in 2 cases, which is $33.3 \%(2 / 6)$;

- type IV - in 4 cases, which is $66.7 \%(4 / 6)$.

4. The occurrence of the quickly bifurcating superior rectal artery - present in $1(2.5 \%)$ case.

- type V.

Types III, IV and V constituted $66.7 \%$ of total cases, where more than one SB occurred; $20 \%$ of total cases, where the quickly bifurcating SB occurred; $66.7 \%$ of total cases, where more than one SRA occurred; and the only case with the occurrence of the quickly bifurcating superior rectal artery which formed type $\mathrm{V}$.

\section{Evaluation of differences in types of IMA branching between sexes}

In Table 1, we presented the distribution of types in general and according to sex. There was no significant difference in types of IMA branching between females and males $(p=0.0853)$.

\section{Evaluation of differences in arterial diameter between sexes}

In women, only the IMA and LCA were significantly narrower than in men (Table 2). 
Table 1. Types of the branching pattern according to sex

\begin{tabular}{lccc}
\hline Type & Females & Males & Total \\
\hline IA & $4(21.05)$ & $6(28.57)$ & 10 \\
IB & $4(21.05)$ & $4(19.05)$ & 8 \\
IC & $3(15.79)$ & $2(9.52)$ & 5 \\
IIA & $1(5.26)$ & $7(33.33)$ & 8 \\
IIB & $2(10.53)$ & $0(0.00)$ & 2 \\
III & $2(10.53)$ & $0(0.00)$ & 2 \\
IV & $2(10.53)$ & $2(9.52)$ & 4 \\
V & $1(5.26)$ & $0(0.00)$ & 1 \\
\hline
\end{tabular}

Table 2. Comparison of arterial diameter between sexes [mm]

\begin{tabular}{lccc}
\hline Type & Females & Males & P \\
\hline Inferior mesenteric artery & $4.02(0.56)$ & $4.58(0.93)$ & 0.0292 \\
Left colic artery & $2.42(0.56)$ & $3.14(0.83)$ & 0.0060 \\
Ascending colic artery & $2.31(0.52)$ & $2.61(0.75)$ & 0.2930 \\
Descending colic artery & $2.09(0.54)$ & $1.97(0.33)$ & 0.5048 \\
Main sigmoid artery & $2.05(0.65)$ & $2.17(0.83)$ & 0.6130 \\
Main superior rectal artery & $2.85(0.68)$ & $3.17(0.91)$ & 0.2222 \\
\hline
\end{tabular}

Evaluation of the relationship between the level of IMA arising from the aorta and type of its branching

The differences were not significant (Table 3).

\section{Evaluation of the relationship between diameters of IMA branches and type of its branching}

Diameters of evaluated arteries according to type of branching are presented in Table 4.

The average diameter of DscCA was different between types ( $p=0.00042$ ). This difference is caused by its significantly smaller diameter in type IA in comparison to type IC and type V.
The mean diameter of the main sigmoid artery was different between types ( $p=0.0007)$. This difference is caused by a significantly smaller diameter in type IA than in type IB, IIA and V and a significantly smaller diameter in type IC in comparison to type IB and also a significantly smaller diameter in type III compared to types IB and V.

The average diameter of the main SRA was also different between the types $(p=0.0003)$. This difference is caused by its significantly smaller diameter in type IA in comparison to types IB, IIA, IIB and V and also a significantly smaller diameter in type IV in comparison to type IIA.

There was no significant difference in the diameter of the LCA ( $p=0.0121)$ and AscCA ( $p=0.0106)$.

The diameter of IMA was reduced after giving rise to the LCA (IMA before branching $4.39 \pm 0.90 \mathrm{~mm}$ vs. IMA after branching $3.64 \pm 0.77 \mathrm{~mm}, \mathrm{p}=0.0001$ ).

\section{Evaluation of the IMA diameter impact on other arteries}

In the correlation analysis, only the diameter of IMA correlated with the diameter of the superior rectal artery (Table 5).

\section{DISCUSSION}

The key value of the present work is that it presents a systematic classification of IMA branching patterns and its origin based on anatomical dissection. It studies the occurrence of additional sigmoidal branches or superior rectal arteries and raises the question whether there are any branching patterns in which we can expect such variations. Furthermore, the research reveals a few cases, which to our best knowledge have not been presented before. It also raises the question of whether the IMA is character-

Table 3. The relationship between the level of inferior mesenteric artery arising from the aorta and type of its branching. Percentage for type is presented in parenthesis $(p=0.7818)$

\begin{tabular}{lccccc}
\hline Type & L2/3 & L3 & L3/L4 & L4 & L5 \\
\hline IA & $5(50 \%)$ & $4(40 \%)$ & $0(0 \%)$ & $1(10 \%)$ & $0(0 \%)$ \\
IB & $0(0 \%)$ & $2(25 \%)$ & $2(25 \%)$ & $3(37.5 \%)$ & $1(12.5 \%)$ \\
IC & $0(0 \%)$ & $0(0 \%)$ & $1(20 \%)$ & $4(80 \%)$ & $0(0 \%)$ \\
IIA & $2(25 \%)$ & $3(37.5 \%)$ & $1(12.5 \%)$ & $2(25 \%)$ & $0(0 \%)$ \\
IIB & $0(0 \%)$ & $0(0 \%)$ & $0(0 \%)$ & $2(100 \%)$ & $0(0 \%)$ \\
III & $1(50 \%)$ & $0(0 \%)$ & $0(0 \%)$ & $1(50 \%)$ & $0(0 \%)$ \\
IV & $1(25 \%)$ & $1(25 \%)$ & $2(50 \%)$ & $0(0 \%)$ & $0(0 \%)$ \\
V & $0(0 \%)$ & $0(0 \%)$ & $0(0 \%)$ & $1(100 \%)$ & $0(0 \%)$ \\
Total & 9 & 10 & 6 & 14 & 1 \\
\hline
\end{tabular}


Table 4. Diameters of arteries according to the branching pattern [mm]

\begin{tabular}{lcccccc}
\hline Type & $\begin{array}{c}\text { Inferior mesenteric } \\
\text { artery }\end{array}$ & $\begin{array}{c}\text { Left colic } \\
\text { artery }\end{array}$ & $\begin{array}{c}\text { Ascending colic } \\
\text { artery }\end{array}$ & $\begin{array}{c}\text { Descending colic } \\
\text { artery }\end{array}$ & $\begin{array}{c}\text { Main sigmoid } \\
\text { artery }\end{array}$ & $\begin{array}{c}\text { Main superior rectal } \\
\text { artery }\end{array}$ \\
\hline IA & $4.14(0.64)$ & $2.44(0.56)$ & $2.07(0.51)$ & $1.73(0.35)$ & $1.56(0.25)$ & $2.20(0.22)$ \\
IB & $4.80(1.28)$ & $3.50(1.08)$ & & & $2.68(0.93)$ & $3.47(0.75)$ \\
IC & $4.76(1.03)$ & & $2.33(0.27)$ & $2.47(0.19)$ & $1.60(0.34)$ & $2.76(0.56)$ \\
IIA & $4.07(0.52)$ & $3.10(0.42)$ & $3.11(0.63)$ & $2.01(0.27)$ & $2.42(0.28)$ & $3.73(0.69)$ \\
IIB & $4.12(0.06)$ & $2.05(0.22)$ & & & $2.84(0.54)$ & $3.80(0.71)$ \\
III & $4.39(0.09)$ & $1.98(0.05)$ & & & $1.53(0.50)$ & $2.90(0.02)$ \\
IV & $3.84(0.12)$ & $2.60(0.47)$ & & & $1.91(0.54)$ & $2.47(0.11)$ \\
V & $3.85(0.00)$ & $2.79(0.00)$ & $2.65(0.00)$ & $2.64(0.00)$ & $3.79(0.00)$ & $4.17(0.00)$ \\
P & 0.3589 & 0.0121 & 0.0106 & 0.0042 & 0.0007 & 0.0003
\end{tabular}

Table 5. Correlations between the diameter of inferior mesenteric artery (IMA) and other arteries

\begin{tabular}{lcc}
\hline Correlation of IMA diameter with & R & P \\
\hline Left colic artery & 0.3239 & 0.0577 \\
Ascending colic artery & 0.0249 & 0.9081 \\
Descending colic artery & 0.1334 & 0.5343 \\
Main sigmoid artery & 0.1682 & 0.2994 \\
Main superior rectal artery & 0.3542 & 0.0250 \\
\hline
\end{tabular}

ised by high morphological variations such as the coeliac trunk.

Our proposed classification for the IMA is numbered from the most frequent to the least, and is divided according to morphology: although type III (5\%) occurred less frequently than type IV (10\%), the branching pattern of type III includes only arteries physiologically posing branches of IMA. In contrast, type IV consists of ascending lumbar artery, which is not a default branch of the IMA.

One of the first classifications was formulated by Testut and Latarjet [25] in the $20^{\text {th }}$ century, and it covered two branching types - type I, separate LCA origin and type II, fan-shaped branching pattern [21]. Patroni et al. [21] used the Latarjete's classification to analyse 113 patients. The present research showed that type I occurred in 80 patients and type II in 33 patients (Table 5 ). In comparison to the Latarjete's classification, type I was found in $60 \%$ of cases and type II in $25 \%$ of cases in our study. A more extensive classification can be found in a study of 115 inferior mesenteric arteries performed by Zebrowski et al. in 1971 [28]. In the specimens they investigated, four forms of the IMA were observed:

- form 1 - the IMA divided into the LCA and common rectal-sigmoid trunk. Available database does not contain the results concerning the frequency of branching pattern types presented in their study;

- form 2 - the IMA divided into the superior rectal and colosigmoid trunk;

- form 3 - the IMA divided into two large arterial trunks: rectal-sigmoid and colosigmoid;

- form 4 - trifurcation type.

The research carried out by Zebrowski et al. [28] widely describes variations in the morphology of IMA and often suggests multiple anastomoses occurring between the majority of secondary branches, which is nowadays considered as a norm in the immediate vicinity of the wall of the large intestine and rectum. There is a strong connection between the classification proposed in 1971 and ours: type I from the present study and form I from the Zebrowski's research are almost the same. Similarly, form 3 (from the Zebrowski's research) and type IV described in our study with the inclusion of the ascending lumbar artery and also form 4 from the previous classification, is identical as our type II. However, the main difference between these classifications is that in our classification types are strictly based on the branching pattern of IMA and subtypes are distinguished based on the morphology of the biggest and in vast majority of cases first branch of the IMA - left colic artery - in contrast to the research done by Zebrowski et al. [28], where sigmoidal branches were used as a differentiating factor. We consider our idea as an innovation and improvement. One of relatively few IMA studies performed on foetuses has been conducted by Nuzhat [15]. A group of 100 cases was subjected to dissection to observe the origin and branching pattern of the IMA. The research has shown that the IMA branched off the abdominal aorta at the level of 
L3 in 99 (99\%) cases and only in 1 (1\%) case at the level of L1. Out of 100 foetuses, 5 foetuses showed a variation - in 1 foetus, the LCA was arising from the abdominal aorta, and in 3 foetuses, the IMA was giving a branch to the left kidney. Ke et al. [11] also have done research on anatomical variations of the IMA and LCA. It was evaluated by three-dimensional (3D) computed tomography angiography (CTA) and performed on 188 patients with abdominal and pelvic contrast-enhanced CT scans, which were retrospectively enrolled and 3D-CTA was reconstructed [11]. However, the proposed classification is different from that by Zebrowski et al. [28]:

- type 1 - the LCA arose independently from the IMA;

- type 2 - the LCA and SB were given off at the same point;

- type 3 - the LCA and sigmoid arteries were branched from a common trunk from the IMA;

- type 4 - the LCA was lacking.

Similarly, as in our study, the LCA was used as a differential factor in describing various types of the branching pattern of IMA. However, there are some key differences. The research performed by Ke et al. [11] was focused on the LCA and sigmoid arteries - the classification does not mention any SRA and its position relative to a branching pattern, which can be seen as a significant feature in our type III, where superior rectal arteries are originating prior to other branches. Type 4 in Ke's study [11] may seem unspecified because the lack of LCA in type 4 can be interpreted as if there was no LCA and its branches and also as absence of LCA where its branches - AscCA and DscCA - are originating directly from IMA (type IC referring to our classification), which can be crucial in planning surgeries like rectal cancer surgery procedures. That research distinguishes only the situation where the level of IMA origin is below the upper border of $L 3$ and cephalic to upper border of $L 3$, while in our study we described precisely the number of vertebrae at which the origin of IMA was observed. Research results described by different authors are presented and compared in the Tables 6 and 7.

The unique origin points of IMA at the level of fifth lumbar vertebra (L5) to our best knowledge have not been reported before and figure as a novelty. Another issue our research is questioning is the evaluation of occurrence of additional sigmoidal branches or superior rectal arteries and their potential connection to the branching pattern.

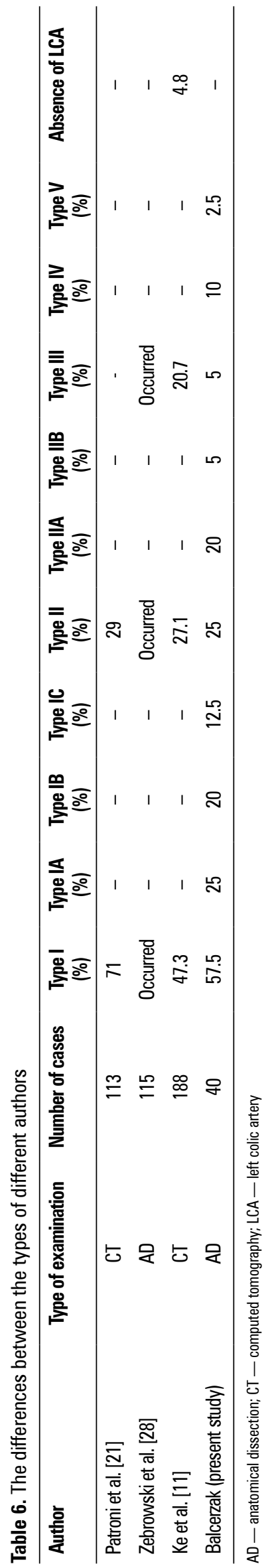


Table 7. Different origin points of inferior mesenteric artery in different studies

\begin{tabular}{lccccccc}
\hline Author & L1 (\%) & L2/L3 (\% & L3 (\%) & L3/L4 (\%) & L4 (\%) & L4/L5 (\%) & L5 (\%) \\
\hline Zebrowski et al. [21] & - & 3.5 & 67 & 20 & 7.8 & 1.7 & - \\
Ke et al. [11] & 88.3 & 88.3 & 88.3 & 11.7 & 11.7 & 11.7 & 11.7 \\
Nuzhat (Foetuses) [15] & 1 & - & 99 & - & - & - & - \\
Balcerzak (present study) & - & 22.5 & 25 & 15 & 35 & - & 2.5 \\
\hline
\end{tabular}

The results presented in this study reveal that in every case where type IV or type V were observed, more than one sigmoidal branch and more than one superior rectal artery occurred. Furthermore, 57.1\% (12/21) of discussed variations occurred in types III, IV, and V. That raises a question whether types IV and $\checkmark$ can be characterised by multiple secondary branching bifurcations. In our opinion, further research is necessary to approve or deny this thesis. The same as for the coeliac trunk, the IMA is a major branch of the abdominal artery and has a vital function in supplying a significant part of the digestive system. However, is IMA characterised by high morphological variations such as the coeliac trunk? The answer to that question is not simple, as the coeliac trunk has been a subject of multiple studies since antiquity, where Galen was the first to describe the distribution of coeliac trunk branches. He concluded during his research that blood supplying the stomach, liver, and spleen comes from another source than the blood supplying the intestines [20]. According to Poynter [22], IMA has not been extensively studied before 1922 and the literature was not very extensive. The artery would appear to be rather stable and not subject to much variation. Cavasse (1856) [6] found an accessory hepatic artery as one of its branches and noted that it occasionally furnishes renal branches. Vicq D'Azyr [see 2] reported the absence of a connection between the middle colic and the left colic arteries. Poynter [22] observed a middle colic artery that arose as a large branch from the left colic near its origin [3]. With the passing of time and the increasing number of tests performed, the object of which was IMA, the number of occurring variations increased: duplication of this artery has been reported. The IMA may arise from the left common iliac artery. Its left colic branch may be absent. The IMA occasionally encroaches on the territory of the superior mesenteric artery, can be replaced by the superior mesenteric artery, or originates from it. In the place of a single inferior mesenteric trunk, two arteries originated from the aorta $2.6 \mathrm{~cm}$ apart, the inferior one located $3 \mathrm{~cm}$ superior to the bifurcation of the aorta. The superior of the two was directed superiorly and gave rise to two branches [3, 12, 15, 27].

Coeliac trunk is surely an artery that shows great variability in large numbers $[20,23,26]$; however, to answer the question whether IMA is characterised by as high morphological variations as the coeliac trunk, in our opinion, there is a need to further analyse and study the IMA branching patterns.

Laparoscopic surgery for colorectal cancer has recently gained wide clinical acceptance. However, due to the narrow view and lack of tactile sensation under laparoscopy, vascular bifurcation and variations could be misidentified and injured, which may cause severe complications, such as massive bleeding and bowel ischaemia [4]. Therefore, it is required to be preoperatively aware of the arterial branching or variations, which are very helpful for surgeons to make pre-operative strategies and perform safe and rapid vessel ligation and lymph node dissection.

Locating the origin of the IMA is also the first key step in rectal cancer surgery.

Many surgeons prefer to perform high ligation of the IMA during rectal cancer surgery to gain complete lymphadenectomy and better mesenteric mobilisation [5, 8].

Low ligation of IMA is recommended to protect blood supply of anastomosis during rectal cancer surgery. However, it increases the operational difficulty under laparoscopy because of the lympho-adipose tissue surrounding the IMA trunk and the highly variant pattern of IMA bifurcation, especially when some dense fibrous tissue surrounding IMA seems like vessel branch $[10,11]$.

If the branching pattern of the IMA trunk and additional secondary branches were preoperatively visualised, these surgical procedures would be much less challenging, but if the visualisation is not possible and surgeons during the procedure notice a branching pattern which can be often related to the occurrence of additional sigmoidal branches or superior rectal arteries, they can react properly, suspect a possible expanded branching connection and gain valuable knowledge resulting in successful operations without any complications. 
Although the subject of this paper has previously been examined in previous studies, our findings highlight some previously unobserved variations. Furthermore, the present study collects, summarizes, and develops the classifications of previous authors, extends them with new types and subtypes, studies the relationships between the IMA branching pattern and the occurrence of additional secondary arterial branches, as well as between this pattern and its level of origin. Moreover, this study takes up the subject of IMA diversity, compares it to the coeliac trunk and provides a clear division between the IMA types and their nomenclature, which seems necessary for all clinicians.

Every research expanding our knowledge about the IMA as well as other major blood vessels is invaluable because it leads us to a better comprehension of how it originates, which are its branching patterns, and when we can suspect any additional branches or arteries and connections between these features, as they provide better outcomes of surgical procedures and increase the quality of life for our patients.

\section{Limitations of the study}

The present study does have some limitations. One of them is the heterogeneous nature of the classification which depends on several morphological details, such as a type of their arising. Due to the possible margin of error resulting from the potential presence of atherosclerotic lesions and related deformations, as well as changes in the direction of the aorta, our analysis of IMA origin points should be taken with caution. Finally, as this is only an anatomical study, a spectrum of variation could be presented; further studies should examine the potential value of angiography or magnetic resonance imaging for this purpose. Nonetheless, this study helps raise awareness of "what and where" to look for, and offers a uniform classification and terminology which can be used as a foundation for communication with surgeons, particularly those harvesting tendons for transplants. The small size of the research sample $(n=40)$ also presents a limitation; even so, this group is larger than used in anatomical studies. In addition, it should be noted that there are significant limitations in the availability of this type of material.

\section{CONCLUSIONS}

The presented classification is based on the major branch of IMA - left colic artery - as the most important differential factor and also on sigmoidal branches as well as the SRA. It consists of detailed specifications of most important bifurcations occurring during the course of IMA: type I in $57.5 \%$ of cases, followed by type II in $25 \%$ of cases, type III in $5 \%$ of cases, type IV in $10 \%$ of cases, and type V, as novelty, in $2.5 \%$ of cases.

The results revealed that there is a significant probability (38.1\%) of the occurrence of additional sigmoidal branches or superior rectal arteries in types III, IV and V.

The study includes a few novelties, which, to our best knowledge, have not been presented before: the case of IMA branching pattern called in our classification as type $V$, which consists of the ascending lumbar artery, two cases of IMA branching pattern called in our classification as type III with two superior rectal arteries originating firstly from the IMA and the case of the unique origin point of IMA from the abdominal artery at the level of fifth lumbar vertebra (L5).

\section{Acknowledgements}

The authors wish to express their gratitude to all those who donated their bodies to medical science.

\section{Conflict of interest: None declared}

\section{REFERENCES}

1. Benton RS, Cotter WB. A hitherto undocumented variation of the inferior mesenteric artery in man. Anat Rec. 1963; 145: 171-173, doi: 10.1002/ar.1091450208, indexed in Pubmed: 13967564.

2. Bergman RA, Afifi AK, Miyauchi R. Anatomy Atlases, An anatomy digital library [WWW Document]. Illus. Encycl. Hum. Anat. Var. 2015.

3. Bergman R, Thompson S, Afifi A, Saadeh F. Compendium of human anatomic variation. Urban \& Schwarzenberg, Baltimore 2018.

4. Bonnet $S$, Berger $A$, Hentati $N$, et al. High tie versus low tie vascular ligation of the inferior mesenteric artery in colorectal cancer surgery: impact on the gain in colon length and implications on the feasibility of anastomoses. Dis Colon Rectum. 2012; 55(5): 515-521, doi: 10.1097/ DCR.0b013e318246f1a2, indexed in Pubmed: 22513429.

5. Boström P, Rutegård J, Haapamäki M, et al. Arterial ligation in anterior resection for rectal cancer: A validation study of the Swedish Colorectal Cancer Registry. Acta Oncol. 2014; 53(7): 892-897, doi: 10.3109/0284186X.2014.913101, indexed in Pubmed: 24954367.

6. Cavasse. Deux anomalies artérielles, cartiods et inferiuer diaphragmatiques. Bulletins et Mem. de la Société Anatomique de Paris. 1856; XXXI(3): 72-73.

7. Chiene. Complete obliteration of the cœliac and mesenteric arteries; the viscera receiving their supply through the extra-peritoneal system of vessels. Am J Med Sci. 1869; 
57(113): 231-232, doi: 10.1097/00000441-18690100000045.

8. Chin CC, Yeh CY, Tang R, et al. The oncologic benefit of high ligation of the inferior mesenteric artery in the surgical treatment of rectal or sigmoid colon cancer. Int J Colorectal Dis. 2008; 23(8): 783-788, doi: 10.1007/s00384-0080465-5, indexed in Pubmed: 18438677.

9. Gwyn DG, Skilton JS. A rare variation of the inferior mesenteric artery in man. Anat Rec. 1966; 156(2): 235-237, doi: 10.1002/ar.1091560211, indexed in Pubmed: 5969677.

10. Horton KM, Fishman EK. CT angiography of the mesenteric circulation. Radiol Clin North Am. 2010; 48(2): 331-345, viii, doi: 10.1016/j.rcl.2010.02.004, indexed in Pubmed: 20609877.

11. Ke J, Cai J, Wen X, et al. Anatomic variations of inferior mesenteric artery and left colic artery evaluated by 3-dimensional CT angiography: Insights into rectal cancer surgery: a retrospective observational study. Int J Surg. 2017; 41: 106-111, doi: 10.1016/j.ijsu.2017.03.012, indexed in Pubmed: 28323157.

12. Kitamura $S$, Nishiguchi $T$, Sakai $A$, et al. Rare case of the inferior mesenteric artery arising from the superior mesenteric artery. Anat Rec. 1987; 217(1): 99-102, doi: 10.1002/ar.1092170113, indexed in Pubmed: 3454570.

13. Kostinovitch LI. A case of simultaneous occurrence of a number of variations of the visceral branches of the abdominal aorta. Anat Rec. 1937; 67(4): 399-403, doi: 10.1002/ar.1090670402.

14. Moore KL, Dalley AF. Clinical Oriented Anatomy, 4th ed. Lippincott Williams \& Wilkins, Philadeplphia 1999.

15. Nuzhat A. Anatomy of inferior mesenteric artery in fetuses. Scientifica (Cairo). 2016; 2016: 5846578, doi: 10.1155/2016/5846578, indexed in Pubmed: 27313956.

16. Olewnik $Ł$. Fibularis tertius: anatomical study and review of the literature. Clin Anat. 2019; 32(8): 1082-1093, doi: 10.1002/ca.23449, indexed in Pubmed: 31408221.

17. Olewnik $\measuredangle$, Łabętowicz $P$, Podgórski $M$, et al. Variations in terminal branches of the popliteal artery: cadaveric study. Surg Radiol Anat. 2019; 41(12): 1473-1482, doi: 10.1007/ s00276-019-02262-3, indexed in Pubmed: 31134299.

18. Olewnik $Ł$, Waśniewska $A$, Polguj $M$, et al. Rare combined variations of renal, suprarenal, phrenic and accessory hepatic arteries. Surg Radiol Anat. 2018; 40(7): 743-748, doi: 10.1007/s00276-018-2026-0, indexed in Pubmed: 29667030

19. Olewnik $\measuredangle$, Wysiadecki G, Polguj $M$, et al. A rare anastomosis between the common hepatic artery and the superior mesenteric artery: a case report. Surg Radiol Anat. 2017; 39(10): 1175-1179, doi: 10.1007/s00276-017-1859-2, indexed in Pubmed: 28432408.

20. Olewnik $九$, Wysiadecki G, Polguj M, et al. Types of coeliac trunk branching including accessory hepatic arteries: a new point of view based on cadaveric study. Folia Morphol. 2017; 76(4): 660-667, doi: 10.5603/FM.a2017.0053, indexed in Pubmed: 28612916.

21. Patroni A, Bonnet S, Bourillon C, et al. Technical difficulties of left colic artery preservation during left colectomy for colon cancer. Surg Radiol Anat. 2016; 38(4): 477-484, doi: 10.1007/ s00276-015-1583-8, indexed in Pubmed: 26526820.

22. Poynter CWM. Congenital anomalies of the arteries and veins of the human body with bibliography. University Studies of the University of Nebraska, 1922 22: 1-106.

23. Pinal-Garcia DF, Nuno-Guzman CM, Gonzalez-Gonzalez ME, et al. The celiac trunk and its anatomical variations: a cadaveric study. J Clin Med Res. 2018; 10(4): 321-329, doi: 10.14740/jocmr3356w, indexed in Pubmed: 29511421.

24. Samura M, Morikage N, Mizoguchi T, et al. Effectiveness of embolization of inferior mesenteric artery to prevent type II endoleak following endovascular aneurysm repair: a review of the literature. Ann Vasc Dis. 2018; 11(3): 259-264, doi: 10.3400/avd.ra.18-00064, indexed in Pubmed: 30402173.

25. Testut L, Latarjet A. Traité d'anatomie humaine. Doin \& Cie, Paris 1949.

26. Torres K, Staśkiewicz G, Denisow M, et al. Anatomical variations of the coeliac trunk in the homogeneous Polish population. Folia Morphol. 2015; 74(1): 93-99, doi: 10.5603/FM.2014.0059, indexed in Pubmed: 25792402.

27. Yi SQ, Li J, Terayama H, et al. A rare case of inferior mesenteric artery arising from the superior mesenteric artery, with a review of the review of the literature. Surg Radiol Anat. 2008; 30(2): 159-165, doi: 10.1007/s00276-0070298-x, indexed in Pubmed: 18189117.

28. Zebrowski W, Augustyniak E, Zajac S. Variation of origin and branches of the inferior mesenteric artery and its anastomoses. Folia Morphol. 1971: 510-517. 